\title{
Vestibular stimulation and primitive reflex integration may drive multisensory processing: putative principles for a Targeted sensorimotor therapy (TSMT)
}

\section{Szilvia Harsanyi}

Debreceni Egyetem

Karoly Dobos

Debreceni Egyetem

\section{Brigitta Tele-Heri}

Debreceni Egyetem

Judit Palinkas

Debreceni Egyetem

Fanni Fenyosi

BHRG Foundation

\section{Csaba E More}

Debreceni Egyetem

Judit Zsuga ( $\square$ zsuga.judit@med.unideb.hu )

University of Debrecen https://orcid.org/0000-0002-5350-8188

\section{Research article}

Keywords: sensorimotor integration, multisensory integration, vestibular stimulation, primitive survival reflex inhibition, TSMT

Posted Date: March 20th, 2020

DOI: https://doi.org/10.21203/rs.3.rs-18167/v1

License: (c) (1) This work is licensed under a Creative Commons Attribution 4.0 International License. Read Full License 


\section{Abstract}

Background: At birth the vestibular system is fully mature, and primitive reflexes are functional whilst higher order sensory processing is yet to develop in the full-term neonate. Sequential motor development driven by primitive survival reflexes sets the appropriate framework for development of sensory processing including multisensory processing and sensorimotor integration.

Results and conclusions: The current paper lays out a putative theoretical framework to account for the role vestibular stimulation may have driving multisensory and sensorimotor integration. Accordingly, vestibular stimulation, by activating the parieto-insular vestibular cortex, and/or the posterior parietal cortex may provide the cortical input for multisensory neurons in the superior colliculus that is needed for multisensory processing. Furthermore, we propose that primitive survival reflex-driven motor development, by inducing change of reference frames, may shape the receptive field of multisensory neurons. This, by leading to lack of spatial contingency between formally contingent stimuli, may cause degradation of prior motor responses, hence lead to integration of reflexes. Integration of primitive survival reflexes is mandatory prerequisite for cortically controlled motor responses to emerge. Additionally, we offer a testable hypothesis explaining the beneficial effect of sensory integration therapies regarding attentional processes. Key concepts of a sensorimotor integration therapy (e.g. targeted sensorimotor therapy (TSMT)) are also put into a neurological context. TSMT utilizes specific tools and instruments. It is administered in 8-weeks long successive treatment regimes, each gradually increasing vestibular and postural stimulation, so sensory-motor integration is facilitated, primitive reflexes are inhibited, and muscle strength is increased. Empirically TSMT is indicated for various diseases. Theoretical foundations of this sensorimotor therapy are discussed.

\section{Background}

The mature neonate is born with a fully functional vestibular organ, and a set of primitive survival reflexes that drive maturation of the central nervous system, psychomotor and cognitive development [1]. While sensory organs develop in utero, higher order sensory processing is yet to mature in the full-term neonate [2]. Sequential motor development driven by primitive survival reflexes sets the appropriate framework for development of sensory processing including multisensory processing $[3,4]$. Multisensory processing, a fundamental mechanism for disambiguating complex environmental signals [5], may contribute to a multitude of diseases and disorders if impaired. Previous reports have established compromised multisensory integration (MSI) in delayed motor development [2], moderately severe to severe cerebral palsy [6, 7], intellectual disability [8, 9], autism spectrum disorder [10, 11], problems with attention including diagnosed attention deficit, hyperactivity disorder [12], sensory organ dysfunction [13], presence of sensory processing disorders [14]. Furthermore, as MSI has been implicated in language development $[15,16]$ and learning disabilities e.g. dyslexia [17].

The current paper lays out a putative theoretical framework to account for the role vestibular stimulation may have driving multisensory and sensorimotor integration. This framework offers insight regarding a 
hypothetical role for multisensory processing in primitive reflex integration, and provides a testable hypothesis explaining the beneficial effect of sensory integration therapies regarding attentional processes. Furthermore, implications of these processes a form of sensorimotor therapy are discussed.

\section{The role of vestibular inputs in the maturation of the nervous system}

The vestibular system is fully operational in the term infant and offers continuous gravitational sensory input that will affect the whole brain [18]. Vestibular sensory perception manifests as a ubiquitous model of gravity in the human brain, which evolves through the interaction with the environment. A fundamental attribute of the vestibular system is compilation of the absolute geocentric, idiothetic reference frame by determining absolute body motion in gravitational space. Input from visual and somatosensory modalities on the other hand contribute to the development of egocentric and allocentric reference frames, respectively [19]. This allows distinguishing movement in the environment from self-movement. Establishing an idiothetic coordinate system encompassing representations of verticality will enable differentiation of spatial relations in a three-dimensional space $[19,20]$. This will form the basis of postural and motor coordination, fine motor control and visual processing [21,22]. As such, vestibular signals may be used to disambiguate conflicting or inaccurate information by reconciling diverse signals $[23,24]$. Hence vestibular function forms the basis of postural and motor coordination, as well as gaze stabilization $[20,25]$. Both linear and rotational input will work to stabilize the head on neck and body, stabilize gaze during active and passive head and body movement [20]. In fact, the vestibular system is instrumental in coordinating head movement with eye movement, offering the foundations for postural tonic control and coordinated control of the eyes $[23,26]$. It is the vestibular system via the vestibuloocular reflex (VOR) that compensates for head rotations above $100 \%$ second, a velocity often encountered during usual daily activities allowing for stabilization of foveal gaze on target [25]. Feedback received regarding the timely and coordinated visual, proprioceptive and vestibular input will constitute the formation of spatial representation both of self and the environment [20]. This constant vestibular backdrop will be used to refine the rudiments of inborn body schema [27] by decoding the multisensory input offered by primitive reflexes [19], and related spatial cognition [19]. Spatial representations are mandatory for higher level cognitive processes such as spatial memory, mental calculations, objectbased mental transformations, social cognition and emotional regulation $[19,20]$. Furthermore, vestibular signals underlie change of perspective from an egocentric to an allocentric viewpoint, by means of visuospatial manipulations. This enables taking third-person perspective mandatory for empathy, understanding and predicting emotions and intentions of others [28]. Spatial memory enables internal simulation and re-representation of the sensory-motor loop's activity in anticipation of future events [20], contributing to a cognitive map possibly used by other processes e.g. model-based reinforcement learning (for an overview see $[29,30]$ ). Conversely patients with vestibular dysfunction have been described to suffer from short-term memory loss, concentration, impaired VOR leading to reading disabilities, impaired ability to estimate basic numeric attributes of the environment, like distances and weights, translating into poor arithmetic skills [19]. Vestibular impairment furthermore can impair self- 
perception and body schema construction. This hinders awareness of body parts, distinction of self from the environment resulting in feelings of depersonalization and out of body projections $[19,20]$.

Vestibular stimulation is a fundamental driver of brain maturation, as several of the primitive survival reflexes utilize vestibular sensory input to elicit the motor responses. These primitive reflexes albeit mandatory for timely development of voluntary motor function must be inhibited giving way to postural reflexes governed by higher centers [25]. During neurotypical development the basic mechanism for inhibiting primitive reflexes is stereotyped repetition of reflex movements [18].

\section{Emergence and integration of primitive survival reflexes during neurodevelopment}

Primitive reflexes are transiently present at birth and are instrumental in the infant's postnatal survival. They are automatic movement patterns, elicited by labyrinth-induced vestibular input in the brainstem [31]. They serve to facilitate mapping of sensory and motor information in the brain and prepare the term infant to move against gravity, thus governing early motor development [1,32]. Primitive survival reflexes become inhibited in an orderly manner, allowing cortically controlled motor responses to emerge $[1,18$, $25,33]$. Thus early presence, followed by programmed inhibition is mandatory for the development of the visual system, posture and higher order processes [25]. Retained primitive reflexes beyond six to twelve months of age signal immaturity of the nervous system and correspond to developmental delays in numerous areas [26]. Retained primitive reflexes impede sensory processing and related sensory-motor integration, laying the groundwork for impaired motoric function, vestibulo-visual integration, underlying visual fixation and saccadic eye movements $[1,26]$. These impairments will contribute to lagging academic achievement as reading and writing ability is impaired, and behavioral disturbances [34]. Hierarchical organization of psychomotor ontogenetic stages becomes evident by developmental delays, or trauma when higher centers become incapacitated liberating primitive reflexes organized at lower levels leading to dysregulated adaptive function [34].

Of the numerous primitive survival reflexes, the Moro reflex, the TLR, the ATNR and the STNR share several attributes in terms of their mechanism and influence on central nervous system maturation [25, 34]. These reflexes are all evoked by vestibular stimulation, and produce motor output utilizing vestibulospinal, vestibulo-cerebellar and vestibulo-ocular loops [26]. Thus, they are instrumental in developing posture, balance and coordination as well as perceptual processing underlying receptive language, reading and writing skill development $[25,31]$.

Moro reflex develops between the $9^{\text {th }}-12^{\text {th }}$ gestational week, and normally becomes inhibited by the $2^{\text {nd }}$ $-4^{\text {th }}$ postnatal month. It may be elicited by sudden loud noises, bright light, change of temperature, or backward drop of the head [32]. As a response, the hands become extended, the infant grasps then crosses the arms in front of the trunk. Inhibition of Moro reflex occurs at the time rolling emerges [32]. Retained Moro reflex can contribute to hypersensitivity to light, sound and temperature that leads to sympathetic overstimulation, heightened arousal, hence providing a continuous backdrop of angst. This on one hand leads to impaired pupillary response to light, poor balance and coordination, on the other 
exhausts cognitive load hindering the ability to categorize and to form perceptual schemas [18, 25, 35]. Hence it may be speculated that retained Moro reflex interferes with deducting statistical regularities underlying co-occurring events, hence delaying the accumulation of experience required to draw statistical inference needed for MSI.

Tonic labyrinth reflex (TLR), closely bound to Moro reflex is fundamental to develop proper alignment of the head and body with respect to its environment. Prone TLR develops in utero and becomes inhibited around the $4^{\text {th }}$ postnatal month [1]. Anteflexion of the infant's head causes flexion of all extremities in front of the body. Supine tonic labyrinth reflex develops at birth, to become gradually inhibited from 6 weeks to 3 years of age. It may be evoked by retroflexion of the head causing extension of the trunk, and the extremities. It offers a rudimentary mechanism to cope with gravity. TLR influences the balance between extensor and flexor muscles and as a result extensor tone develops in a rostro-caudal direction. This will enable the position of the head to be stabilized against gravity, hence the reference point for assessing space, distance and velocity will emerge $[18,26]$. If retained, the TLR interferes with the development of postural reflexes especially the ocular and the head righting reaction. Resultant reciprocal disturbance between the vestibular and visual system will impair oculomotor development and balance, rendering the idiothetic reference frame improper and hindering spatial judgement [25]. Furthermore, lack of sufficient head control will hinder the development of eye-hand control, accommodation and convergence, all mandatory for visual acuity and coordination $[18,25]$.

Asymmetric tonic neck reflex (ATNR) emerges on the $18^{\text {th }}$ gestational week and becomes integrated between the $3^{\text {rd }}-9^{\text {th }}$ months. Turning the head to the right side causes the extension of right the hand and leg paralleled by the flexion of the extremities on the opposite side and vice versa. ATNR splits movements of the two sides of the body vertically. Its inhibition is mandatory to allow coordinating the two sides as the body prepares for interweaving movement patterns seen with creeping, walking, running [35]. It is instrumental for visual development, as similarly to TLR it contributes to eye-hand control. Failure of its inhibition interferes with left-right integration [1], development of dominance regarding hand, foot and eye [25]. Furthermore, it hinders the eyes ability to cross the vertical midline, a skill mandatory for reading and writing with the latter skill further burdened by poor eye-hand control $[1,26]$. Retained ATNR interferes with convergence as symmetrical release of eyes is impaired upon focusing from near to far [35] and leads to poor eye tracking [1]. Symmetric tonic neck reflex (STNR) is a reflex that emerges parallel to the inhibition of TLR [18]. Some theorize that it is the modification of TLR seen during its inhibition, as response to the extension of the head will lead partial modification of TLR, by flexing and not extending the lower extremities [18]. It surfaces after the ATNR is inhibited, hence by this time the ability to coordinating the two sides of the body across the vertical midline is possible, and the eyes focus to the distance $[26,35]$.

STNR emerges postnatally between the age of $6^{\text {th }}-9^{\text {th }}$ months, to become inhibited between $9^{\text {th }}-11^{\text {th }}$ months of age. If elicited in the quadrupedal position by anteflexion of the head, flexion of the arms and extension of the legs ensues, while retroflexion of the head leads to extension of the arms and flexion of the legs $[1,36]$. The STNR will enable the body to move against gravity, facilitates focusing to near 
distance and for the first time permits the body to lift. This promotes integration of vestibular, visual and proprioceptive input during movement [35]. Integration of STNR is instrumental to coordinate movements along the horizontal midline, therefore STNR has major contribution to posture and coordination $[25,26]$. Delayed STNR integration will contribute to predominant flexor tone, leading to poor posture, especially evident when sitting [26]. Furthermore, adjustment of visual focus between different distances will be impaired leading to poor hand-eye coordination, manifesting in clumsiness and impaired ability to copy graphical elements [25]. Albeit STNR lifts the body, movement is not yet possible, therefore its delayed inhibition will impair cross-pattern creeping [18]. Primitive survival reflexes are illustrated in Figure 1.

\section{Results}

\section{Development of multisensory integration}

MSI is a process for combining sensory information obtained from distinct sensory channels on the level of the single neuron. Albeit ubiquitous, and evolutionarily long-standing, it is not coded in the genome. Its maturation shows a protracted course during postnatal life [37-39]. Multisensory maturation is driven by experience that enables linking cross-modal attributes of self-referential and environmental events to each other. Hence sensory experiences form the basis for developing a world model based on perceived cross-modal contingencies [37]. The vestibular system offers a basic example for resolving sensory ambiguity by means of MSI yielding novel sensory representations, to differentiate between voluntary actions and environmental perturbations [40]. Conversely, MSI based on integration of sensory information weighted according to the precision of sensory cues was shown to underlie optimal motion and orientation estimates when sensorimotor integration was investigated using a limb movement control task [41]. Corroborating evidence was further provided in terms of sensorimotor postural control where dynamic reweighting of sensory, sensory-orientation cues explained behavior [42].

The brain is adept to function in noisy environments, where sensory signals vary in terms of their intensity, relative timing and spatial location as behavior must be governed despite uncertainties and unexpected perturbations [5]. One approach for the brain to cope with the multitude of signals, is abstracting distinct yet related sensory attributes by dedicated multisensory neurons [23]. By combining independent estimates of unimodal sensory input, noise may be reduced parallel to increased precision, as complementary or redundant signals are generally integrated optimally (for a review see [2]). Computational theories depicting the combination of unisensory attributes of sensory cues for multisensory processing utilize Bayesian decision theory models [43-45]. This posits that the brain decodes hidden environmental regularities by means of statistical inference, and as such computes the probability distributions of prior knowledge and estimates the likelihood of sensory information [5]. Multisensory processing is consistent Bayesian with principles that describe perception rooted in MSI, and sensorimotor behavior [46].

A further implication of interpreting MSI under the premises of Bayesian decision theory models is that perception may be regarded as inference about the causes of sensory cues, and attention may be 
conceptualized as precision of environmental and self-referential sensory signals [37, 47]. Hence attention manifests by weighting data with respect to estimated precision [48]. Furthermore, assuming that the only unexplained sensory details of the environment are prediction errors, attention derived weights reflect estimated precision and manifest biologically as synaptic gain [48]. Hence multisensory processing serves to optimize precision-weighted prediction errors. This concept allows explaining how multisensory experience enhances the salience of events if stimuli fulfill certain conditions for temporal synchrony, spatial congruence and stimulus efficacy $[5,49,50]$. Corroborating evidence shows correlated activity of unisensory afferents of the anterior ectosylvian sulcus (AES) and their respective multisensory postsynaptic common target neurons in the superior colliculus (SC) as evidences shows that here synaptic weights are adjusted in line with Hebbian learning rules [37]. Summarizing, it would follow that intersensory redundancy derived from concurrent presentation of the same information over several modalities, by increasing precision of the multimodal percept would lead to heightened attention.

Given that MSI serves to craft a model of the environment that is specific for the individual's cross-modal experiences, MSI develops if different sensory signals are in close temporal and spatial correspondence, possibly indicating their probable joint origin from common events [37]. Conversely MSI is more pronounced if the relationship between stimuli is in line with prior expectations or congruent with natural interrelation of senses [38]. Temporal congruence of two stimuli mandates that distinct modalities reach the target multisensory convergence area within a temporal window that is able to adjust for different architectural delays inherent of said sensory modalities [51]. For example tactile latencies are on average $40 \mathrm{~ms}$ shorter than visual latencies in the posterior parietal cortex (PPC) [49] and visual cues precede auditive ones [52]. Additionally, amodal properties like temporal synchrony, common rhythm and spectral information between cross-modal stimuli facilitate integration. This process is fundamental for infants acquiring speech, as intrinsic rhythmic regularities and temporal correlation between facial and lip movements and auditory input drives MSI for perceptual processing needed for speech [52, 53]. Congruent audiovisual stimuli presenting the same speech input was shown to improve accuracy of recognition when compared to audio or visual stimulus administered alone [54].

A central property for integrating multisensory information is reliability, that translates into computations as weights that are re-iterated online for adaptive behavior. Multisensory neurons have overlapping receptive fields that are responsive to their unisensory input at birth, but their ability to integrate multisensory input is the result of sensorimotor experience-driven maturation [39]. Accordingly, the ability for MSI is not innate, it needs to be learnt $[39,55]$. For example, previous works have shown that statistically optimal integration of haptic and visual information for postural control develops gradually, as it is insufficient in 8-year-old [56], but approximates adult maturity in 10-year-old children [57]. Concordant to these findings, is the suboptimal integration of cues for navigation in children younger than 8 years of age [56]. Cuturi and Gori [24] have shown that vision and touch are both influenced by the same vestibular/proprioceptive priors regarding head and body orientation with respect to gravity. Furthermore, children as young as 6 years of age showed some level of optimal statistical inference in a simple position-estimation Bayesian task, where data was best fitted by Bayesian model fit. These findings enabled the authors to conclude that some level of Bayesian integration is already present in 
early childhood, which is gradually refined parallel to acquiring experience, as the brain learns to approximate [46]. Alternatively, cross-sensory calibration may be the antecedent of MSI, allowing the more accurate sense to teach the others. For example the haptic system may calibrate vision, while the visual system may calibrate hearing for assessing space and hearing seems to calibrate vision with respect to time perception [2].

Signal efficacy also has some implications regarding MSI. Inverse effectiveness, a universal phenomenon described in context of multisensory enhancement is the ability of weak sensory signals to elicit a superadditive response, if cross-modal stimuli are coincident in time and space and fall on overlapping multisensory receptive fields [5,58]. Increasing signal saliency decreases the efficacy for multisensory enhancement as unisensory responses are already elaborate [49]. Hence the ability to detect subthreshold cross-modal stimuli may be explained be the law of inverse effectiveness and superadditivity [59]. For example, temporal regularity and cross-modal temporal cueing, properties indicative of the congruency condition for MSI were shown to lower auditory thresholds reflected by improved auditory perception in response to subthreshold auditory responses [52]. Additional to speech perception benefiting from temporal correlation of visual cues in relation to audition, facilitation of perception was further shown with regards to other combinations of subthreshold stimuli including olfactory and visual modalities in Drosophila [38]. Taking the principle of dual coding into consideration, e.g. that if information is processed through multiple senses, limited processing capabilities may be circumvented, it would follow that interventions facilitating multisensory learning may help overcoming difficulties stemming from impairment of one or more sensory organs.

Evidence shows that multisensory neurons are present at birth on various areas of the brain, dedicated to multisensory processing, including SC, the parieto-insular vestibular cortex (PIVC), and PPC [58, 60, 61]. General concepts of MSI are derived from the canonical model, the cat SC, as this structure has abundance of multisensory neurons and may be conveniently studied under experimental conditions. Preclinical studies show that although SC neurons are responsive to more than one sensory modality at birth [37, 58], they are incapable of synthetizing cross-modal stimuli, therefore generation of novel unitary behavior is absent [58]. This is dependent on ipsilateral corticotectal inference received from the association cortex (the AES and the rostral lateral suprasylvian sulcus in particular) that develops 4 to 12 weeks after birth [61]. That influence from ipsilateral association cortex is a prerequisite for MSI was evidenced by different paradigms. Reversible, chronic 4 to 8 weeks long pharmacological deactivation of the association cortex in early-life, rendered the association cortex insensitive to cross-modal experiences. Delay of timely acquisition of sensory experiences required for SC maturation caused protracted delay in maturation of MSI up to 1.5 to 4 years [62]. Conversely, selective deactivation of this area impaired SC multisensory neurons' ability to integrate cross-modal inputs underlying MSI [63]. Further indirect evidence regarding the permissive effect of the association cortex with respect to the functional capacity of SC multisensory circuit is the correlated activity elicited by cross-modal cues in the SC multisensory neurons and their unisensory cortical afferents [63]. Hence the association cortex may serve as a portal that allows adaption to specific experience-based multisensory experience via altering the functional capability of SC multisensory circuit [64]. In fact, albeit an environment devoid of early cross-modal 
challenge hinders SC's ability to integrate converging sensory inputs, this activity does re-emerge if crossmodal experience is obtained later life [63]. Previous reports showed that dark-reared cats were able to reestablish their capacity for MSI of paired visuo-auditory stimuli in a month after being exposed to weekly visuo-auditory stimulation. This suggests that SC multisensory neurons retain considerable plasticity during later stages of development allowing adaptation [63]. This implicates the possibility for synergistic beneficial effects between sensory integration therapies and corrective measures for sensory organs (e.g. correcting visual acuity and hearing), as the tailored sequences of exercises rich in invariant cross-modal features along with enhanced sensory perception of sensory stimuli could facilitate multisensory learning.

Although the homologue area of cat AEC is not known in primates [58], we point to two candidate multisensory cortical areas that may assume a role similar to the cat AES in terms of MSI. The first one is the primate PIVC+, an area possibly made up of two distinct anatomical and functional subareas, the posterior insular cortex (PIC) and PIVC [60]. PIC is a visuo-vestibular area, while PIVC is a multisensory cortical region with neurons responsive to vestibular (angular acceleration), somatosensory (e.g. stimulation of neck and shoulder mechanoceptors while the head is stable) and visual (especially optokinetic) stimuli $[60,65]$. PIVC is considered a hub for vestibular cortex and there is some evidence for a human homologue area as well $[66,67]$. The second one is the PPC responsible for transforming sensory signals into reference frames that participate in guiding gaze or reach [58]. Some parts (e.g. the ventral intraparietal area) were shown to receive direct vestibular projections, hence they are considered to be part of the vestibular cortical network [68]. Furthermore, using horseradish peroxidase retrograde labeling, corticotectal cells were identified in both the parieto-insular vestibular cortex and the posterior parietal cortex [69]. These findings together tempt the speculative notion vestibular stimulation may enhance maturation of PIVC and the PPC (Figure 2). Consequently, PIVC and/or PPC will via their corticotectal afferents may facilitate MSI in the SC. This putative mechanism may be compromised in preterm infants constrained to incubators and those needing prolonged hospitalization after birth, thus explaining later seemingly unexplained developmental delays [70-72]. The findings of Weinstein and colleagues [73] showing deviant sensory-motor reactivity in preterm children paralleled by disturbed integrity and lagging maturational level of early and late maturing fiber tracts further support this notion, given the significance of early experiences and need for intact corticotectal projections [37].

Benefits of MSI are relevant if they manifest in behavioral output like superadditivity or subadditivity, a nonlinear combination of modality-specific influences. Pronounced enhancement superseding the sum of individual neuronal responses may ensue under conditions of MSI if two unimodal stimuli fall within overlapping excitatory receptive fields. With this regard spatial congruence of stimuli is fundamental to allow co-activation of multisensory areas by unisensory modalities. During development this evolves by sculpting of spatially overlapping receptive fields from their larger neonatal templates, based on early experiences [37]. This process is substantial from infancy as compiling sensory experiences from birth will underlie the brain's ability to model the world using Bayesian inference. Conversely, sensory representations of multisensory areas e.g. the SC, the PPC, are topographically organized and show spatial correspondence since multisensory neurons have multiple excitatory receptive field, one for each 
modality they respond to [58]. For example, the receptive field for visual and auditory modalities of an audiovisual neuron would correspond, if both the visual and auditory receptive fields would be for example in the nasal space [61]. Hence a cross-modal activation with respect to a single event will activate the same multisensory neuron. Many excitatory receptive fields are however bordered by an inhibitory region. Therefore, if spatial location of cues becomes disparate so activation from a modality falls on this inhibitory border zone it will inhibit the activating effect of the other modality, causing depressed neuronal response, and consequently degrades performance [58]. Furthermore, if one of the sensory cues is outside of the receptive field, the integrated response will be absent [61]. To adhere to this very robust spatial principle of MSI while enabling independent movement of each sensory organ, modality-specific receptive fields may be linked to eye position a phenomenon described in the SC and the PPC [74-76]. Hence this oculocentric coordinate frame enables adjustment of visual, auditory, somatosensory or cross-modal goals with respect to the orientation of gaze, yielding a unified coherent locus of activity within the sensory-motor map [58]. However, intermediate reference frames, receptive fields that shift only partially in response to the position of the eyes were also described previously [58]. These were implicated to enable transformation between distinct coordinate frames, for example from eye to head or to body centered frames $[77,78]$. Transitioning from a head centered to a body centered reference frame is mandatory to interpret the relationship between body motion and vestibular stimulation. For example, PIVC, the multisensory vestibular cortical area, was shown to have a reference frame that is intermediate between head and body centered and showed weak modulation by eye position [79]. Similarly, intermediate reference frames for head and body centered movement were reported in the cerebellum [23] in an area containing bimodal neurons that integrate vestibular and cervical proprioceptive inputs. Brooks and colleagues have further shown that in adult monkeys with intact vestibulospinal reflexes, proprioceptive and vestibular signals cancel each other out when the head is passively moved relative to a stationary body [80], a maneuver known to elicit ATNR [35]. Misalignment of receptive fields thus may hinder cancellation of different signals, possibly allowing for a maladaptive motoric response, possibly even retained primitive reflexes.

Starting from these considerations we put forward yet another highly speculative notion that one possible mechanism contributing to primitive reflex inhibition is related to multisensory processing (Figure 2). Namely respective receptive fields underlying the cross-modal attributes of primitive reflexes will delineate in a way that coincident sensory cues will fall on either the inhibitory marginal zone of the previously joint receptive fields, or outside of it. Hypothetically this may lead to degradation of the primitive reflex profile. This is likely if one considers the natural course of reflex integration is through repetition e.g. the ATNR is integrated by crawling, the STNR by rocking on hands and knees etc. [81]. Moreover, there is further evidence regarding the ability of standardized repetition of stereotyped infant movements to inhibit ATNR [82]. Observations further depict that neonatal MSI develops more rapidly if multisensory stimuli are presented with invariant cross-modal features, showing limited ambiguity in a controlled setting, as opposed to the general rearing conditions where relative signal intensities, timing, and spatial alignment as well as competing cues cause considerable ambiguity [63]. It was also shown that dark-reared cats were able to re-establish their capacity for MSI of paired visuo-auditory stimuli in a month after being 
exposed to weekly visuo-auditory stimulation, suggesting that SC multisensory neurons retain considerable plasticity during later stages of development allowing adaptation [63].

\section{Sensorimotor integration in postural development}

Postural stability, e.g. providing sustained control of the unstable, multisegmental body in the face of instability, rests on neural integration of sensory input and contingent sensorimotor integration $[55,83]$. Accordingly signals of all relevant sensory (visual, vestibular, proprioceptive) modalities are combined with motor efference signals reflective of the net of prior motor experiences to optimize posture [84, 85]. A key component of postural adaptation is feedback offered via fast and slow feedback loops, corresponding to reflexive and intentional control, respectively [83]. The fast loop acting through spinal, brainstem and transcortical reflexes responds to environmental perturbation with a very low latency (50$100 \mathrm{~ms}$ ). These reflexes include the vestibulospinal reflex that coordinates movement of the head and neck with that of the trunk [21], the cervico-collic reflex that stabilizes the head on the trunk and the vestibulo-collic reflex that stabilizes the head in space [85] and transcortical reflexes. Transcortical reflexes are triggered by multimodal sensory stimuli and delivered irrespective of consequences. They are further influenced by input from the cerebellum, posterior parietal and frontal cortex, hence may be modulated by external instructions, behavioral context, including task goals [83]. Nevertheless, transcortical reflexes are reflexive in the sense that the automatic motor responses reflect environmental casualty derived from past choices.

The slow loop on the other hand utilizes central selection and optimization pathways to enable online planning, selection and inhibition of non-selected alternatives, with a variable latency of 180-500 ms [83]. To maintain appropriate posture, MSI continuously readjusts weights of visual, vestibular and proprioceptive information, thus more and less reliable sensory stimuli are up- and downregulated, respectively [39]. For example, de-emphasizing visual cues leads to greater reliance on proprioceptive and vestibular input to maintain postural control. The ability to reweight incoming sensory signals develops after birth. Initially young infants rely mainly on vision, as the ability to reweight visual and touch sensory information emerges at around 4 years of age [86], and it is between the ages of 9 to 12 years that all three sensory modalities (including vestibular cues) feed into the multisensory inputs for postural control $[39,84]$. Ability to reweight information gradually decreases optic flow induced perturbations, a process that parallels accumulation of upright posture experience [55]. Prior research has shown that sensorimotor control of human posture becomes adaptive a few months after obtaining independent sitting [55], however, multisensory fusion, e.g. intermodal reweighting of coexisting modalities by combining sensory information in a statistically optimal way, emerges gradually and reaches maturity around the age of 10 years [39]. This is in line with the finding that tasks that challenge postural control by providing conflicting sensory information, are difficult to perform before mid-childhood [84].

The fundamental influence gross motor development has with respect to postural sensorimotor integration should be noted at this point, as the reference frame used for balance and posture changes during development. For example, for infants who are at the stage when independent movement is yet 
not possible, the head serves as the reference frame as initial postural responses mature along a cephalocaudal gradient. Appropriate control emerges first in the muscles of the neck, then in the trunk followed by the legs [22]. Development of head control is the first signpost for postural equilibrium. At this developmental stage moving visual stimuli will evoke postural responses [87], and head and gaze stabilization underlie the reference frame for reaching, allowing visual cues to predominate control of postural equilibrium [22]. These processes are governed by the proper evolution and integration of primitive survival reflexes [26, 34]. Later, as quadrupedal and upright stance becomes possible, the frame of reference for balance equilibrium changes so the supporting surface will be the reference frame [22], and proprioception will become the primary source of somatosensory information used for controlling posture [88]. Accordingly, touch information at the fingertips was shown to provide information regarding body orientation and becomes embodied in the body schema [89]. Thus, it would follow that change of reference frames for postural control, by changing receptive fields of multisensory neurons may contribute to the gradual achievement of optimal adult-like neural computations to estimate postural orientation.

\section{Discussion}

\section{Putative neurological foundations of a novel sensorimotor integration therapy}

Targeted sensorimotor therapy (TSMT) is a form of sensorimotor integration therapy developed in Hungary. Albeit over twenty years have passed since its initial introduction, only limited evidence is available regarding its efficacy. A small clinical trial involving 25 preschoolers (aged 5 years old) diagnosed with attention deficit and hyperactivity disorder underwent individual TSMT for one year to assess the beneficial effects of TSMT. During this year, 90 minutes long therapies were administered twice a week by a single therapist. Children were assessed by the Condition and Movement Test (CMT) at baseline and during follow-up. This test is validated to measure neurophysiological maturity [90-92]. It assesses five domains including primitive reflexes, motoric-control, body schema perception/spatial orientation/ dexterity, tactile sensory system and rhythm. Scores are expressed as a percentage, with lower scores reflecting impairment, with values lower than $50 \%$ indicating significant development delays, whereas values exceeding $75 \%$ reflecting typical neurodevelopment and maturity. Moreover, higher scores were shown to predict better school performance reflected by better grades [93]. At 12 months follow-up the children were 6 years old. Measures of central nervous system maturity showed significant improvement reflected by changes in scores for primitive reflex profile (mean at baseline $21.42 \pm 8.74 \%$ vs. mean at follow-up $50.71 \pm 12.41 \% \mathrm{p}<0.001$ ), motoric-control (mean at baseline $17.5 \pm 9.02 \%$ vs. mean at follow-up $45.75 \pm 13.10 \% \mathrm{p}<0.001$ ), body schema, spatial orientation, and dexterity (mean at baseline $39.23 \pm 8.10 \%$ vs. mean at follow-up $77.23 \pm 11.76 \% \mathrm{p}<0.001$ ), tactile sensory system (mean at baseline $42.5 \pm 12.31 \%$ vs. mean at follow-up $68.21 \pm 7.34 \%$ p $<0.001$ ), rhythm (mean at baseline $7.2 \pm 8.42 \%$ vs. mean at follow-up $35.6 \pm 8.69 \% \mathrm{p}<0.001)$. Furthermore, results have shown that after one year of TSMT therapy several domains of neurodevelopment (e.g. motoric control, body schema perception and tactility) became comparable to that observed in typically developing 6-year-old preschoolers [91]. A successive case report has also described the beneficial effect of TSMT in a 30 months old boy who 
showed significant developmental delay (delayed movement and language development, lack of voluntary bowel/bladder control, sleep problems). Following 26 weeks of TSMT therapy the child's development significantly improved, he began speaking a few words (mom, mamma, papa and other imitative words), and domains of self-regulation also showed improvement [94]. Empirically, TSMT may be indicated for varying diseases and disorders including delayed motor development, delayed language development, moderately severe to severe cerebral palsy, mental retardation, problems with attention including diagnosed attention deficit, hyperactivity disorder, sensory organ dysfunction, presence of sensory processing disorders. Children having learning difficulties, high risk for dyslexia, dysgraphia, dyscalculia as well as those with behavioral and social difficulties including impulsivity, poor task compliance, aggression, lack of cooperation or autism spectrum disorder may also benefit [94].

The goal of TSMT is facilitation of MSI and sensorimotor learning and increasing attention span. This is achieved by developing acoustic, vestibular, proprioceptive, tactile and visual modalities, adaptive motor responses by delivering stereotyped sequences of exercises rich in invariant cross-modal features. The cornerstones of TSMT are vestibular and multisensory stimulation, integration of retained primitive reflexes, and facilitation adequate postural control. We propose that efficacy of TSMT comes from the strong vestibular stimulation. This by activating the parieto-insular vestibular cortex, and/or the posterior parietal cortex may provide the cortical input for multisensory neurons in the superior colliculus that is needed for multisensory processing. Furthermore, we suggest that intersensory redundancy derived from concurrent presentation of the same information over several modalities, by increasing precision of the multimodal percept may be the reason for improving attention.

TSMT is based on several fundamental principles. It is a regressive therapy as it strongly builds on the most archaic system the vestibular system to facilitate ontogeny of adaptive behavior. It targets the integration of retained primitive reflexes and utilizes the natural course of gross motor development to correct body schema perception, postural instability and facilitate automatic locomotion. A further fundamental attribute is the exploitation of rhythm, audio-visual-motor synchronization, and the vestibulo-ocular reflex. As opposed to other sensory integration therapies (e.g. Ayres therapy) the training program is targeted, as treatment regimes are individualized based on the findings of a detailed physical examination (e.g. CMT, described above). This includes a rudimentary assessment of vision, hearing, muscle tone and hearing allowing early diagnosis and indication of corrective measures (hearing aids, glasses etc.).

The recommended age for TSMT is between 6 months to 12 years of age. TSMT utilizes specific sensory tools (colorful beanbags, balls, etc.) and instruments, e.g. large fitness balls, skateboards, rotating chair, and specialized tilting board. The later are crafted to aid the administration of vestibular stimulation via both the semicircular labyrinth and the otoliths and facilitate MSI underlying development of adaptive postural control.

TSMT is administered in successive 8-weeks long treatment regimens (a sample treatment regime is illustrated in Figure 3). Each regime builds on the preceding one, gradually increasing vestibular and 
postural stimulation. The sequence of exercises is determined by the therapist on a case-by-case basis. As vestibular stimulation is a fundamental component of TSMT regimes, usually the first therapeutic regime contains the highest proportion of passive vestibular exercises. These are generally administered at the beginning of the therapeutic sessions as vestibular stimulation is known to induce arousal via direct projections of the vestibular nuclei to locus ceruleus [95]. Passive vestibular exercises include tasks in which the head's position relative to the body is stationary, rotated to either side, flexed or extended. Active vestibular stimulation includes tumbling, and active exercises on the skateboard. Linear (translational) and rotational acceleration are also delivered using different sensory-motor equipment (balls, skateboard, tilting board etc.). Strong vestibular stimuli are administered by exercises in upsidedown position, tumbling, swinging. Different static postures (e.g. supine, prone, hands and knees, sitting, erect), automatic (e.g. rolling, quadrupedal locomotion- creeping, crawling) and goal directed motoric patterns (e.g. spider, crab and giant walking, bear crawling) are implemented via passive and active exercises.

TSMT encompasses several approaches to inhibit primitive survival reflexes. Treatment regimens strongly rely on the proper execution of ontogenetic movement patterns, specific human motor patterns that emerge at distinct neurodevelopmental stages, e.g. segmental rolling, creeping, crawling, and sitting [96]. Repetitive, stereotyped movements focusing on voluntary inhibition of primitive reflex movements are also used vastly. This may be facilitated by use of beanbags held under the chin, or between a tilted head and ipsilateral shoulder while performing exercises targeting the shoulder grid (pushups, tilt board, tumbling). To inhibit primitive reflex responses by voluntary control, instructions to perform certain manipulations with tools e.g. to hold a beanbag under the chin, atop the head, may be given. Furthermore, as stereotyped movements embedded in an attentive learning context were shown to lead dedifferentiation of cortical representation of sensory inputs [97], it is possible that the repetitive exercises aiming to elicit the retained primitive reflex responses further facilitate reflex integration by inducing plastic changes that lead to altered cortical modulatory input in the brainstem.

As TSMT is fundamentally a sensory-motor integration therapy, motor planning of voluntary movement is also challenged. Exercises for ipsilateral or contralateral synchronization of extremities, cross-patterns and serial movement patterns consisting of two to eight sequential movements are also fundamental in TSMT. Development of repetitive movement patterns is usually accompanied by nursery rhymes chanted by both the therapist (parent) and possibly the child, in a way that chanting is aligned to the rhythm of the exercises. In fact, the motto of TSMT is that if a therapy is silent it is not TSMT. Hence TSMT utilizes coadministration of stimuli that share congruent amodal properties. Instructions are given for verbal tasks synchronously with the rhythm of a motor task for example when bouncing on the fitness ball, using the balance board, jumping, quadrupedal locomotion etc. to facilitate verbal-motor synchronization. During therapy, the child has the ability to observe the face of the therapist (parent) while talking to allow accumulation of experience mandatory for speech acquisition.

Correction of posture by reeducation, may be facilitated by either providing verbal instruction or offering educational manipulations. Nonetheless in order to correct postural responses facilitated by the fast 
feedback loop, training should implement measures that enable interference from the slow feedback loop [83]. Well established therapeutic interventions, for example hippotherapy also utilize varying, slowly delivered sensory stimuli to facilitate improvements of postural control [98]. The tilting board used by TSMT may be instrumental in driving such slow postural corrective measures. It may be used in a quadrupedal, sitting, kneeling and standing position. It can be used to induce tilting in the antero-posterior and lateral directions. The usual frequency is of tilting is between $0.5-1 \mathrm{~Hz}$. This timeframe supplemented by verbal instructions and physical corrective measures may enable the slow intentional loop to step in and re-educate the incorrect postural responses delivered by the fast loop. Additionally, by placing small beanbags for example on top of the head, sensory information about the head's orientation in space is augmented so voluntary attempts to improve head control are facilitated. Finally, using the board for lateral tilts in the quadrupedal position offers strong stimuli for inhibiting tonic neck reflexes, an effect that may be further augmented by manipulating the position of the head. Instructions are given so the therapist faces the child and if possible, maintains eye contact while assisting with the exercises. This facilitates understanding by allowing visual access to the face, also assists receptive language development.

\section{Conclusions}

The current article overviews relevant neuroscientific findings and offers novel insight into mechanisms of MSI. It we propose that primitive survival reflex-driven motor development, by inducing change of reference frames, can shape the receptive field of multisensory neurons. This, by leading to lack of spatial contingency between formally contingent stimuli, causes degradation of former motor responses, hence integration of reflexes. Additionally, we propose that vestibular stimulation, by activating the parietoinsular vestibular cortex, and/or the posterior parietal cortex may provide the cortical input for multisensory neurons in the superior colliculus that is needed for multisensory processing. These mechanisms may be instrumental in sensorimotor integration therapies, nonetheless larger clinical studies are needed.

\section{Methods}

The aim of the study was to overview core neurological processes that drive maturation and development of the central nervous system. The theoretical advancements laid out provide a putative mechanism explaining how vestibular stimulation may facilitate MSI in the SC and how MSI may contribute to the integration of primitive survival reflexes. The merit of this concept is that it gives rise the several testable hypotheses carrying clinical and pedagogical implications. The current work builds on former experimental and clinical work of our group and others cited throughout the text, allowing theory formation.

\section{Abbreviations}


anterior ectosylvian sulcus

ATNR

asymmetric tonic neck reflex

CMT

Condition and Movement Test

MSI

multisensory integration

PIC

posterior insular cortex

PIVC

parieto-insular vestibular cortex

PPC

posterior parietal cortex

SC

superior colliculus

STNR

symmetric tonic neck reflex

TLR

tonic labyrinth reflex

TSMT

targeted sensorimotor therapy

\section{Declarations}

Ethics approval and consent to participate

Not applicable

Consent for publication

Not applicable

Availability of data and materials

Not applicable

Competing interests

None of the authors have any conflict of interest.

\section{Funding}


This study was supported by the European Union [EFOP-3.6.2-16-2017-00009 "Establishing Thematic Scientific and Cooperation Network for Clinical Research" (providing support for JZ) and EFOP-3.6.1-162016-00022 „Debrecen Venture Catapult Program” (providing support for SH)], by the New National Excellence Program of the Ministry of Human Capacities (in Hungary) [ÚNKP-18-DE-457 (providing support for JZ)], by the Hungarian Brain Research Program 2.0 [2017-1.2.1-NKP-2017-00002 (providing support for JZ)], and by the János Bolyai Research Fellowship of the Hungarian Academy of Sciences (providing support for JZ).

\section{Authors' contributions}

KD and SH wrote the first draft of the manuscript. KD, SH, JP and FF participated in the collection and interpretation of the relevant literature. CEM and JZ elaborated the theoretical concept. JZ completed the manuscript. All authors read and approved the final manuscript.

\section{Acknowledgements}

Planned sensorimotor therapy is a propriety therapy developed by occupational therapist Katalin Lakatos Ph.D. She worked it out based on her own observations and systematic analysis of data from over 1000 children she personally treated. Currently the therapy is delivered by certified TSMT therapists trained by the BHRG Foundation.

The authors would like to acknowledge Noémi Balogh for the artwork presented in Figure 1.

\section{References}

1 Gieysztor, E.Z. et al. Persistence of primitive reflexes and associated motor problems in healthy preschool children. Arch. Med. Sci. 2018;14:167-173

2 Gori, M. Multisensory Integration and Calibration in Children and Adults with and without Sensory and Motor Disabilities. Multisens Res. 2015;28:71-99

3 Lane, S.J. et al. Neural Foundations of Ayres Sensory Integration. Brain Sci. 2019; doi:10.3390/brainsci9070153

4 Roley, S.S. et al. Understanding Ayres' Sensory Integration. OT Practice 2007;12(17):CE-1-CE-7

5 Chandrasekaran, C. Computational principles and models of multisensory integration. Curr. Opin. Neurobiol. 2017; 43, 25-34

6 Purpura, G. et al. Visuo-haptic transfer for object recognition in children with periventricular leukomalacia and bilateral cerebral palsy. Child. Neuropsychol. 2019;25:1084-1097

7 Pavao, S.L. and Rocha, N.A.C.F. Sensory processing disorders in children with cerebral palsy. Infant. Behav. Dev. 2017;46:1-6 
8 Jarvinen, A. et al. Relations between social-perceptual ability in multi- and unisensory contexts, autonomic reactivity, and social functioning in individuals with Williams syndrome. Neuropsychologia 2015;73:127-140

9 Zhou, H.Y. et al. Multisensory temporal binding window in autism spectrum disorders and schizophrenia spectrum disorders: A systematic review and meta-analysis. Neurosci. Biobehav. Rev. 2018;86:66-76

10 Stevenson, R.A. et al. Multisensory speech perception in autism spectrum disorder: From phoneme to whole-word perception. Autism Res. 2017;10:1280-1290

11 Feldman, J.I. et al. Audiovisual multisensory integration in individuals with autism spectrum disorder: A systematic review and meta-analysis. Neurosci. Biobehav. Rev. 2018;95:220-234

12 Lane, S.J. and Reynolds, S. Sensory Over-Responsivity as an Added Dimension in ADHD. Front. Integr. Neurosci. 2019;13:40

13 Myers, M.H. et al. A pilot investigation of audiovisual processing and multisensory integration in patients with inherited retinal dystrophies. BMC Ophthalmol. 2017;17:240

14 Chang, Y.S. et al. Autism and sensory processing disorders: shared white matter disruption in sensory pathways but divergent connectivity in social-emotional pathways. PLoS One 2014;9:e103038

15 Kumar, G.V. et al. Large Scale Functional Brain Networks Underlying Temporal Integration of AudioVisual Speech Perception: An EEG Study. Front. Psychol. 2016;7:1558

16 Tiippana, K. et al. Multisensory and sensorimotor interactions in speech perception. Front. Psychol. $2015 ; 6: 458$

17 Hahn, N. et al. Impairments of multisensory integration and cross-sensory learning as pathways to dyslexia. Neurosci. Biobehav. Rev. 2014;47:384-392

18 Goddard, S. The role of primitive survival reflexes an the development of the visual system. Mind Moves Institute 1995;6:31-35

19 Le Gall, A. et al. The critical role of vestibular graviception during cognitivo-motor development. Behav. Brain Res. 2019;372:112040

20 Wiener-Vacher, S.R. et al. Vestibular activity and cognitive development in children: perspectives. Front. Integr. Neurosci. 2013;7:92

21 Cullen, K.E. The vestibular system: multimodal integration and encoding of self-motion for motor control. Trends Neurosci. 2012;35:185-196 
22 Assaiante, C. Development of locomotor balance control in healthy children. Neurosci. Biobehav. Rev. 1998;22:527-532

23 Green, A.M. and Angelaki, D.E. Multisensory integration: resolving sensory ambiguities to build novel representations. Curr. Opin. Neurobiol. 2010;20:353-360

24 Cuturi, L.F. and Gori, M. Biases in the Visual and Haptic Subjective Vertical Reveal the Role of Proprioceptive/Vestibular Priors in Child Development. Front. Neurol. 2019;9:1151

25 Gonzalez, S.R. et al. The Correlation between Primitive Reflexes and Saccadic Eye Movements in 5th Grade Children with Teacher-Reported Reading Problems. Optometry \& Vision Development 2008;39:140145

26 Blythe, S.G. Releasing educational potential through movement: A summary of individual studies carried out using the INPP test battery and developmental exercise programme for use in schools with children with special needs. Child Care in Practice 2005;11:415-432

27 Rochat, P. Self-Unity as Ground Zero of Learning and Development. Front. Psychol. 2019;10:414

28 Deroualle, D. and Lopez, C. Toward a vestibular contribution to social cognition. Front. Integr. Neurosci. 2014;8:16

29 Zsuga, J. et al. The proactive model of learning: Integrative framework for model-free and modelbased reinforcement learning utilizing the associative learning-based proactive brain concept. Behav. Neurosci. 2016;130:6

30 Zsuga, J. et al. 'Proactive' use of cue-context congruence for building reinforcement learning's reward function. BMC Neurosci. 2016;17:70

31 Wahlberg, T. and Ireland, D. Can replicating primary reflex movements improve reading ability? Optometry \& Vision Development 2005;36:89-91

32 Marquis, P.J. et al. Retention of primitive reflexes and delayed motor development in very low birth weight infants. J. Dev. Behav. Pediatr. 1984;5:124-126

33 Woollacott, M.H. and Shumway-Cook, A. Changes in posture control across the life span - a systems approach. Phys. Ther. 1990;70:799-807

34 Konicarova, J. et al. Persisting primitive reflexes in medication-naive girls with attention-deficit and hyperactivity disorder. Neuropsychiatr. Dis. Treat. 2013;9:1457-1461

35 Berne, S.A. The Primitive Reflexes: Considerations in the Infant. Optometry \& Vision Development 2006;37:139-146 
36 Taylor, M. et al. Primitive Reflexes and Attention-Deficit/Hyperactivity Disorder: Developmental Origins of Classroom Dysfunction. International Journal of Special Education 2004;19:23-37

37 Wallace, M.T. and Stein, B.E. Early experience determines how the senses will interact. J. Neurophysiol. 2007;97:921-926

38 Shams, L. and Seitz, A.R. Benefits of multisensory learning. Trends Cogn. Sci. 2008;12:411-417

39 Bair, W.N. et al. Development of multisensory reweighting is impaired for quiet stance control in children with developmental coordination disorder (DCD). PLoS One 2012;7:e40932

40 Gutfreund, Y. et al. Gated visual input to the central auditory system. Science 2002;297:1556-1559

41 Sabes, P.N. Sensory integration for reaching: models of optimality in the context of behavior and the underlying neural circuits. Prog. Brain Res. 2011;191:195-209

42 Peterka, R.J. and Loughlin, P.J. Dynamic regulation of sensorimotor integration in human postural control. J. Neurophysiol. 2004;91:410-423

43 Alais, D. and Burr, D. The ventriloquist effect results from near-optimal bimodal integration. Curr. Biol. 2004; $14: 257-262$

44 Kording, K.P. and Wolpert, D.M. Bayesian integration in sensorimotor learning. Nature 2004;427:244247

45 Kording, K.P. et al. Causal inference in multisensory perception. PLoS One 2007;2:e943

46 Chambers, C. et al. The development of Bayesian integration in sensorimotor estimation. J. Vis. 2018;18:8

47 Feldman, H. and Friston, K.J. Attention, uncertainty, and free-energy. Front. Hum. Neurosci. 2010;4:215 48 Feldman, H. and Friston, K.J. Attention, uncertainty, and free-energy. Front. Hum. Neurosci. 2010;4:215 49 Avillac, M. et al. Multisensory integration in the ventral intraparietal area of the macaque monkey. $J$. Neurosci. 2007;27:1922-1932

50 Stanford, T.R. and Stein, B.E. Superadditivity in multisensory integration: putting the computation in context. Neuroreport 2007;18:787-792

51 Harrar, V. et al. Multisensory integration is independent of perceived simultaneity. Exp. Brain Res. 2017;235:763-775

52 ten Oever, S. et al. Rhythmicity and cross-modal temporal cues facilitate detection. Neuropsychologia 2014;63:43-50 
53 Bahrick, L.E. and Lickliter, R. Intersensory redundancy guides attentional selectivity and perceptual learning in infancy. Dev. Psychol. 2000;36:190-201

54 Brown, V.A. and Strand, J.F. "Paying" attention to audiovisual speech: Do incongruent stimuli incur greater costs? Atten Percept. Psychophys. 2019;81:1743-1756

55 Chen, L.C. et al. Development of adaptive sensorimotor control in infant sitting posture. Gait Posture 2016;45:157-163

56 Nardini, M. et al. Development of cue integration in human navigation. Curr. Biol. 2008;18:689-693

57 Gori, M. et al. Young children do not integrate visual and haptic form information. Curr. Biol. 2008;18:694-698

58 Stein, B.E. and Stanford, T.R. Multisensory integration: current issues from the perspective of the single neuron. Nat. Rev. Neurosci. 2008;9:255-266

59 Stevenson, R.A. et al. Superadditive BOLD activation in superior temporal sulcus with threshold nonspeech objects. Exp. Brain Res. 2007;179:85-95

60 Frank, S.M. and Greenlee, M.W. The parieto-insular vestibular cortex in humans: more than a single area? J. Neurophysiol. 2018;120:1438-1450

61 Wilkinson, L.K. et al. The role of anterior ectosylvian cortex in cross-modality orientation and approach behavior. Exp. Brain Res. 1996;112:1-10

62 Rowland, B.A. et al. Brief cortical deactivation early in life has long-lasting effects on multisensory behavior. J. Neurosci. 2014;34:7198-7202

63 Yu, L. et al. Multisensory Plasticity in Superior Colliculus Neurons is Mediated by Association Cortex. Cereb. Cortex 2016;26:1130-1137

$64 \mathrm{Xu}, \mathrm{J}$. et al. Incorporating cross-modal statistics in the development and maintenance of multisensory integration. J. Neurosci. 2012;32:2287-2298

65 Grusser, O.J. et al. Localization and responses of neurones in the parieto-insular vestibular cortex of awake monkeys (Macaca fascicularis). J. Physiol. 1990;430:537-557

66 Gunny, R. and Yousry, T.A. Imaging anatomy of the vestibular and visual systems. Curr. Opin. Neurol. 2007;20:3-11

67 Ventre-Dominey, J. Vestibular function in the temporal and parietal cortex: distinct velocity and inertial processing pathways. Front. Integr. Neurosci. 2014;8:53 
$68 \mathrm{Klam}, \mathrm{F}$. and Graf, W. Vestibular signals of posterior parietal cortex neurons during active and passive head movements in macaque monkeys. Ann. N. Y. Acad. Sci. 2003;1004:271-282

69 Fries, W. Cortical projections to the superior colliculus in the macaque monkey: a retrograde study using horseradish peroxidase. J. Comp. Neurol. 1984;230:55-76

70 Ryckman, J. et al. Sensory processing disorder in preterm infants during early childhood and relationships to early neurobehavior. Early Hum. Dev. 2017;113:18-22

71 Pekcetin, S. et al. The Efficiency of Sensory Integration Interventions in Preterm Infants. Percept. Mot. Skills 2016;123:411-423

72 Rahkonen, P. et al. Atypical sensory processing is common in extremely low gestational age children. Acta Paediatr. 2015;104:522-528

73 Weinstein, M. et al. Neonatal neuropsychology: emerging relations of neonatal sensory-motor responses to white matter integrity. Neuropsychologia 2014;62:209-219

74 Groh, J.M. and Sparks, D.L. Saccades to somatosensory targets. III. eye-position-dependent somatosensory activity in primate superior colliculus. J. Neurophysiol. 1996;75:439-453

75 Hartline, P.H. et al. Effects of eye position on auditory localization and neural representation of space in superior colliculus of cats. Exp. Brain Res. 1995;104:402-408

76 Stricanne, B. et al. Eye-centered, head-centered, and intermediate coding of remembered sound locations in area LIP. J. Neurophysiol. 1996;76:2071-2076

77 Pouget, A. et al. A computational perspective on the neural basis of multisensory spatial representations. Nat. Rev. Neurosci. 2002;3:741-747

78 Snyder, L.H. Frame-up. Focus on "eye-centered, head-centered, and complex coding of visual and auditory targets in the intraparietal sulcus". J. Neurophysiol. 2005;94:2259-2260

79 Chen, X. et al. Diverse spatial reference frames of vestibular signals in parietal cortex. Neuron 2013;80:1310-1321

80 Brooks, J.X. and Cullen, K.E. Multimodal integration in rostral fastigial nucleus provides an estimate of body movement. J. Neurosci. 2009;29:10499-10511

81 Niklasson, M. et al. Sensorimotor therapy: using stereotypic movements and vestibular stimulation to increase sensorimotor proficiency of children with attentional and motor difficulties. Percept. Mot. Skills 2009;108:643-669

$82 \mathrm{McPhillips,} \mathrm{M.} \mathrm{et} \mathrm{al.} \mathrm{Effects} \mathrm{of} \mathrm{replicating} \mathrm{primary-reflex} \mathrm{movements} \mathrm{on} \mathrm{specific} \mathrm{reading} \mathrm{difficulties} \mathrm{in}$ children: a randomised, double-blind, controlled trial. Lancet 2000;355:537-541 
83 Loram, I.D. Postural control and sensorimotor integration. In: Grieve's Modern Musculoskeletal Physiotherapy (Jull, J. et al, ed.), 2015; pp. 4-1-4-14, Elsevier

84 Sa, C.D.S.C. et al. Development of postural control and maturation of sensory systems in children of different ages a cross-sectional study. Braz J. Phys. Ther. 2018;22:70-76

85 Bronstein, A.M. Multisensory integration in balance control. Handb. Clin. Neurol. 2016;137:57-66

86 Bair, W.N. et al. Development of multisensory reweighting for posture control in children. Exp. Brain Res. 2007;183:435-446

87 Peterka, R.J. and Black, F.O. Age-related changes in human posture control: sensory organization tests. J. Vestib. Res. 1990;1:73-85

88 Riley, M.A. et al. Common effects of touch and vision on postural parameters. Exp. Brain Res. 1997;117:165-170

89 Bair, W.N. et al. Children with developmental coordination disorder benefit from using vision in combination with touch information for quiet standing. Gait Posture 2011;34:183-190

90 Lakatos, K. Állapot és mozgásvizsgálat (Condition and movement tests). 2006; BHRG Alapítvány

91 Lakatos, K. A mozgásérettség vizsgálatának jelentősége a tanulási zavarok korai felismerésében (Significance of motor development in early diagnosis of learning difficulties). 2005; PhD Dissertation

92 Heri, B. Az idegrendszeri éretlenség gyakoriságának vizsgálata viselkedés- és magatartászavaros gyerekek körében (Assessment of CNS maturity in children with behavioral disabilities). 2017; MSc in Complex Rehabilitation Thesis

93 Fenyosi, F. Socio-economic status and human capital development. 2014; Thesis for Master of Science in Rural Development

94 Bodor, E. Az organikus érési zavar felismerésea és kezelése TSMT-I terápiával (Early diagnosis and therapy of organic developmental delay using TSMT-I therapy). 2014; BSC Thesis for Physiotherapists 95 Gurvich, C. et al. Vestibular insights into cognition and psychiatry. Brain Res. 2013;1537:244-259 96 Berenyi, M. et al. Phylo- and ontogenetic aspects of erect posture and walking in developmental neurology. Ideggyogy Sz. 2011;64:239-247

97 Byl, N.N. et al. A primate genesis model of focal dystonia and repetitive strain injury: I. Learninginduced dedifferentiation of the representation of the hand in the primary somatosensory cortex in adult monkeys. Neurology 1996;47:508-520 
98 Zadnikar, M. and Kastrin, A. Effects of hippotherapy and therapeutic horseback riding on postural control or balance in children with cerebral palsy: a meta-analysis. Dev. Med. Child Neurol. 2011;53:684691

\section{Figures}

A.

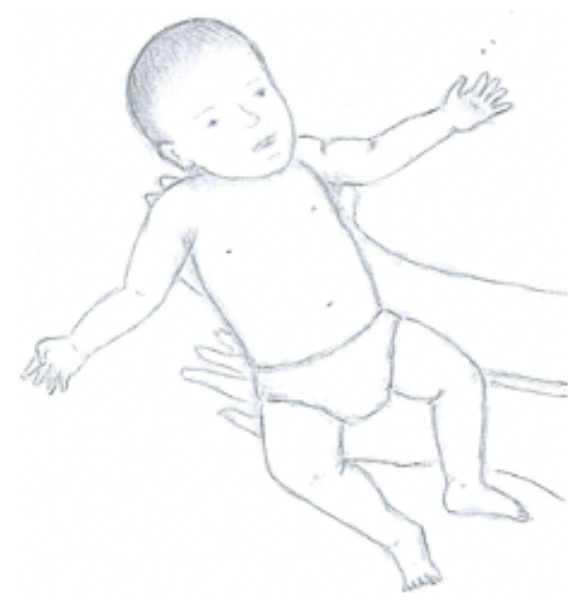

D.

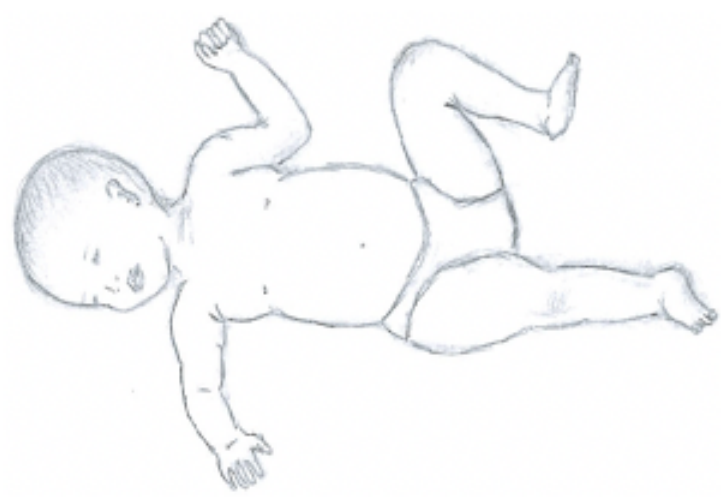

B.

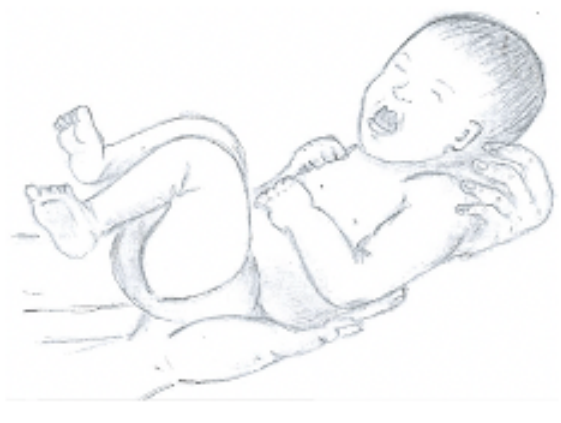

E.

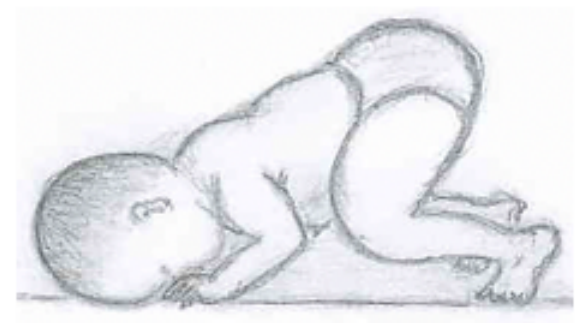

C.

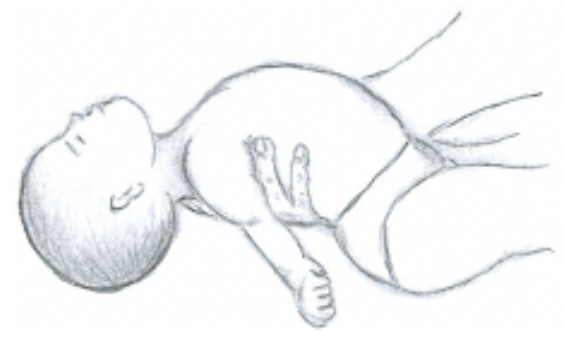

F.

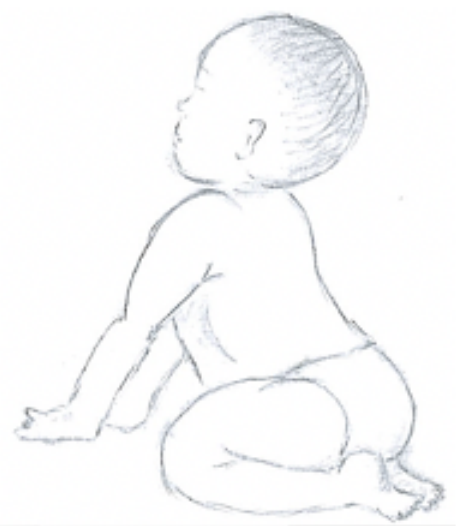

\section{Figure 1}

Illustration of primitive survival reflexes (A: Moro reflex; B: Prone tonic labyrinth reflex; C: Supine tonic labyrinth reflex; D: Asymmetric tonic neck reflex; E: Symmetric tonic neck reflex-anteflexion of the head; F: Symmetric tonic neck reflex-retroflexion of the head) 


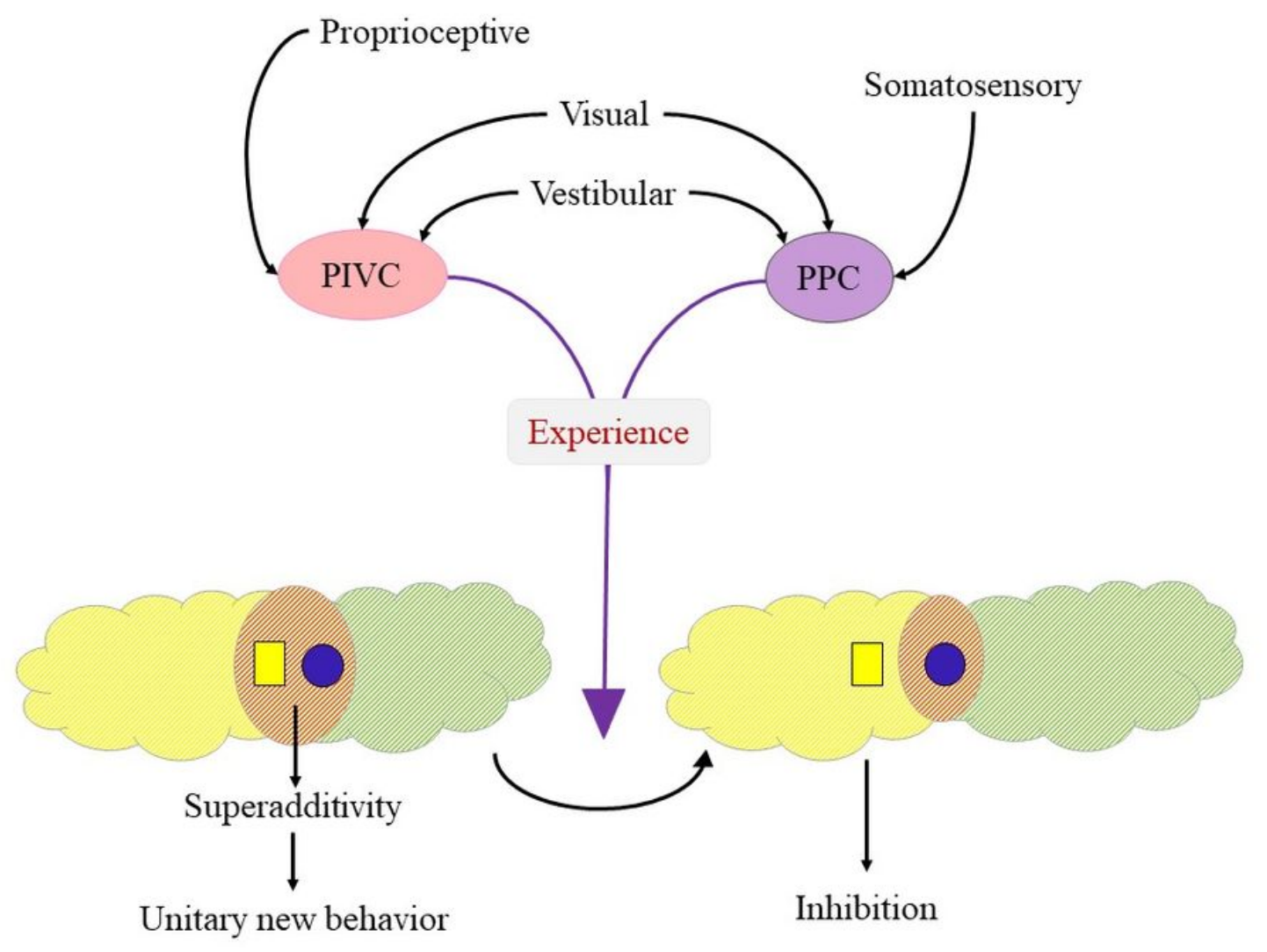

\section{Figure 2}

Putative mechanism for vestibular stimuli-driven maturation of MSI. Sensory stimulation activates higher level multisensory vestibular centers (PIVC and PPC). This on one hand provides sufficient input for maturation of multisensory neurons in the superior colliculus. On the other stimulation of the PPC allows change of reference frames and concurrent alteration of receptive fields of SC multisensory neurons. This experience related change may modify the response given to sensory stimuli from superadditive unitary output (illustrated on the left side) to inhibition (illustrated on the right side). This change in the receptive fields is hypothesized to offer a mechanism for inhibiting primitive reflexes. This hypothetical mechanism may contribute to the efficacy of TSMT. PIVC: parieto-insular vestibular cortex; PPC: posterior parietal cortex 


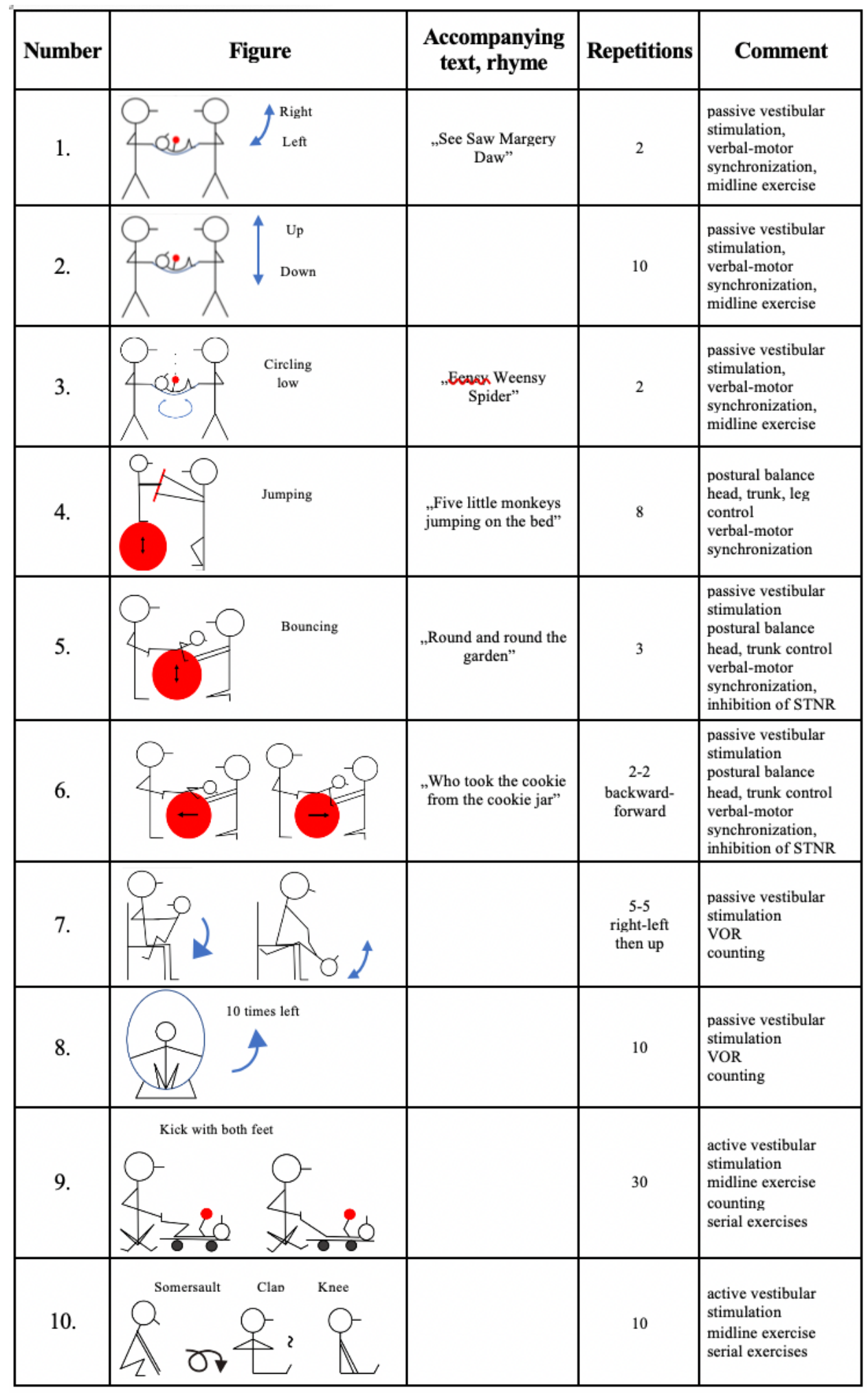

\section{Figure 3}

Illustration of an individualized TSMT regime 